\title{
QUARTIC POLYNOMIALS WITH A GIVEN DISCRIMINANT
}

\author{
JiŘí KLAŠKA \\ Dedicated to the eminent Czechoslovak mathematician Ladislav Skula
}

(Communicated by Milan Paštéka)

\begin{abstract}
Let $0 \neq D \in \mathbb{Z}$ and let $Q_{D}$ be the set of all monic quartic polynomials $x^{4}+a x^{3}+b x^{2}+$ $c x+d \in \mathbb{Z}[x]$ with the discriminant equal to $D$. In this paper we will devise a method for determining the set $Q_{D}$. Our method is strongly related to the theory of integral points on elliptic curves. The well-known Mordell's equation plays an important role as well in our considerations. Finally, some new conjectures will be included inspired by extensive calculations on a computer.
\end{abstract}

\section{(C) 2022}

al Institute

Slovak Academy of Sciences

\section{Introduction}

Let $0 \neq D \in \mathbb{Z}$ and let

$$
Q_{D}=\left\{f(x)=x^{4}+a x^{3}+b x^{2}+c x+d \in \mathbb{Z}[x] ; D_{f}=D\right\}
$$

where

$$
\begin{aligned}
D_{f}= & a^{2} b^{2} c^{2}-4 a^{2} b^{3} d-4 a^{3} c^{3}+18 a^{3} b c d-27 a^{4} d^{2}-4 b^{3} c^{2} \\
& +16 b^{4} d+18 a b c^{3}-80 a b^{2} c d-6 a^{2} c^{2} d+144 a^{2} b d^{2} \\
& -27 c^{4}+144 b c^{2} d-128 b^{2} d^{2}-192 a c d^{2}+256 d^{3}
\end{aligned}
$$

is the discriminant of $f(x)$. In this paper, the set $Q_{D}$ will be studied in detail. Most of the focus will be given to the problem of determining all polynomials in $Q_{D}$. Clearly, this is equivalent to finding all integer solutions of the Diophantine equation $D_{f}=D$. In proving the main results, the following two known theorems will be needed.

Theorem 1.1 (Mordell, 1920). For any given $0 \neq k \in \mathbb{Z}$, the equation

$$
Y^{2}=X^{3}+k
$$

has at most finitely many integer solutions.

Equation 1.3 is often called Mordell's equation, in honour of the contribution Louis Joel Mordell 17 has made to this subject. An extension to Theorem 1.1 was later made by Carl Ludwig Siegel [18. In its simplest form, Siegel's result can be formulated as follows:

Theоrem 1.2 (Siegel, 1929). Let $\alpha, \beta \in \mathbb{Z}$ be such that $4 \alpha^{3}+27 \beta^{2} \neq 0$. Then the equation

$$
\eta^{2}=\xi^{3}+\alpha \xi+\beta
$$

has at most finitely many integer solutions.

2020 Mathematics Subject Classification: Primary 11D25, 11D45, 11 Y50.

Keywords: Quartic polynomial, discriminant, Mordell's equation, elliptic curve. 
There is a standard method for computing all integer solutions of 1.3 and (1.4) using David's bounds and lattice reduction. This method can be found, for example, in [19. At present, this method is implemented in several computer algebra packages, including Magma and Pari (Sage).

Remark 1.3. Mordell's equation has had a long history. First discoveries concerning (1.3) were given in Dickson [2 pp. 533-539] going back to the work of Bachet from 1621. Many interesting historical notes to $(1.3)$ can be found in $[1,5,7,16$. Perhaps the most extensive historical comments related to Mordell's contribution to (1.3) can be found in the recent paper 6].

Throughout this paper, the following notation will be adopted. If $A$ is a finite set, $\# A$ denotes the number of elements of $A$.

\section{Equivalence on the set $Q_{D}$}

Let $f(x)=x^{4}+a x^{3}+b x^{2}+c x+d \in \mathbb{Z}[x]$ and let $D_{f}$ be the discriminant of $f(x)$. Next, let $r_{f}(x)=f(x-a / 4)$. Then

$$
r_{f}(x)=x^{4}+A x^{2}+B x+C \in \mathbb{Q}[x]
$$

where

$$
A=b-\frac{3 a^{2}}{8}, \quad B=c-\frac{a b}{2}+\frac{a^{3}}{8}, \quad C=d-\frac{a c}{4}+\frac{a^{2} b}{16}-\frac{3 a^{4}}{256} .
$$

Moreover, we have

$$
D_{r_{f}}=D_{f}=16 A^{4} C-4 A^{3} B^{2}-27 B^{4}-128 A^{2} C^{2}+144 A B^{2} C+256 C^{3} .
$$

From $(2.2)$, it follows that there exist $R, S, T \in \mathbb{Z}$ such that

$$
A=\frac{R}{8}, \quad B=\frac{S}{8}, \quad C=\frac{T}{256},
$$

where

$$
R=8 b-3 a^{2}, \quad S=8 c-4 a b+a^{3}, \quad T=256 d-64 a c+16 a^{2} b-3 a^{4} .
$$

Hence, we can write 2.1) in the form

$$
r_{f}(x)=x^{4}+\frac{R}{8} x^{2}+\frac{S}{8} x+\frac{T}{256} \in \mathbb{Q}[x] \quad \text { with } \quad R, S, T \in \mathbb{Z} .
$$

We start with a more general theorem.

Theorem 2.1. Let $n \in \mathbb{N}, n \geq 2,0 \neq D \in \mathbb{Z}$ and let

$$
f(x)=x^{n}+a_{n-1} x^{n-1}+\cdots+a_{1} x+a_{0} \in \mathbb{Z}[x]
$$

be an arbitrary polynomial with the discriminant equal to $D$. Further, for any $w \in \mathbb{Z}$, let

$$
f_{w}(x)=\sum_{k=0}^{n} \frac{f^{(k)}(w)}{k !} x^{k},
$$

where $f^{(k)}(w)$ denotes the $k$-th derivative of $f(x)$ at $w$. Then $f_{w}(x) \in \mathbb{Z}[x]$ and all polynomials in $\left\{f_{w}(x) ; w \in \mathbb{Z}\right\}$ have the same discriminant equal to $D$. 


\section{QUARTIC POLYNOMIALS WITH A GIVEN DISCRIMINANT}

P r o of. First, by induction on $k$, it can be proved that $k ! \mid f^{(k)}(w)$ for any $k \in\{0,1,2, \ldots\}$. Hence, $f_{w}(x) \in \mathbb{Z}[x]$. Further, Taylor's theorem yields

$$
f(x)=\sum_{k=0}^{n} \frac{f^{(k)}(w)}{k !}(x-w)^{k} \quad \text { for any } w \in \mathbb{Z} .
$$

Let $\alpha_{1}, \ldots, \alpha_{n}$ be the roots of $f(x)$ in the set of complex numbers $\mathbb{C}$. Then

$$
D=\prod_{i=1}^{n-1} \prod_{j=i+1}^{n}\left(\alpha_{j}-\alpha_{i}\right)^{2}
$$

Next, by (2.8), for any $\alpha \in\left\{\alpha_{1}, \ldots, \alpha_{n}\right\}$, we have

$$
f(\alpha)=\sum_{k=0}^{n} \frac{f^{(k)}(w)}{k !}(\alpha-w)^{k}=0 .
$$

Combining (2.7) with 2.9), we get $f_{w}(\alpha-w)=0$, and thus,

$$
\beta_{1}=\alpha_{1}-w, \ldots, \beta_{n}=\alpha_{n}-w
$$

are the roots of $f_{w}(x)$ in $\mathbb{C}$. Using $(2.10)$, we now get

$$
D_{f_{w}}=\prod_{i=1}^{n-1} \prod_{j=i+1}^{n}\left(\beta_{j}-\beta_{i}\right)^{2}=\prod_{i=1}^{n-1} \prod_{j=i+1}^{n}\left(\alpha_{j}-w-\left(\alpha_{i}-w\right)\right)^{2}=\prod_{i=1}^{n-1} \prod_{j=i+1}^{n}\left(\alpha_{j}-\alpha_{i}\right)^{2}=D,
$$

as desired.

Remark 2.2. Observe that, in Theorem 2.1 $f(x)=f_{0}(x)$. Hence, $f(x) \in\left\{f_{w}(x) ; w \in \mathbb{Z}\right\}$.

Corollary 2.3. Let $0 \neq D \in \mathbb{Z}$ and let $f(x)=x^{4}+a x^{3}+b x^{2}+c x+d \in Q_{D}$. Further, for any $w \in \mathbb{Z}$, let

$$
f_{w}(x)=x^{4}+\frac{f^{\prime \prime \prime}(w)}{3 !} x^{3}+\frac{f^{\prime \prime}(w)}{2 !} x^{2}+\frac{f^{\prime}(w)}{1 !} x+f(w) .
$$

Then (i) and (ii) hold:

(i) $Q_{D}$ is an infinite set and $\left\{f_{w}(x) ; w \in \mathbb{Z}\right\} \subseteq Q_{D}$.

(ii) For any $w \in \mathbb{Z}$, we have $r_{f_{w}}(x)=r_{f}(x)=x^{4}+A x^{2}+B x+C \in \mathbb{Q}[x]$, where $A, B, C$ satisfy (2.2).

Pro of. Part (i) of Corollary 2.3 is a direct consequence of Theorem 2.1 for $n=4$. Part (ii) can be verified by direct calculation.

Lemma 2.4. Let $0 \neq D \in \mathbb{Z}$ and let $f(x), g(x) \in Q_{D}$. Then (i), (ii) and (iii) are equivalent:

(i) There exists $w \in \mathbb{Z}$ satisfying $g(x)=f(x+w)$.

(ii) There exists $w \in \mathbb{Z}$ satisfying $g(x)=f_{w}(x)$.

(iii) $r_{f}(x)=r_{g}(x)$.

Proof. Let $f(x)=x^{4}+a x^{3}+b x^{2}+c x+d, g(x)=x^{4}+\bar{a} x^{3}+\bar{b} x^{2}+\bar{c} x+\bar{d} \in Q_{D}$.

First we show that (i) is equivalent to (ii). Using Taylor's theorem, we obtain

$$
f(x)=(x-w)^{4}+\frac{f^{\prime \prime \prime}(w)}{3 !}(x-w)^{3}+\frac{f^{\prime \prime}(w)}{2 !}(x-w)^{2}+\frac{f^{\prime}(w)}{1 !}(x-w)+f(w)
$$

for any $w \in \mathbb{Z}$. Therefore,

$$
f(x+w)=x^{4}+\frac{f^{\prime \prime \prime}(w)}{3 !} x^{3}+\frac{f^{\prime \prime}(w)}{2 !} x^{2}+\frac{f^{\prime}(w)}{1 !} x+f(w) .
$$


Combining (2.12) with (2.11), we get $f(x+w)=f_{w}(x)$. Hence, (i) and (ii) are equivalent.

Further we prove that (i) is equivalent to (iii). Assume that $g(x)=f(x+w)$ for some $w \in \mathbb{Z}$. Then $(2.12)$ yields

$g(x)=x^{4}+(4 w+a) x^{3}+\left(6 w^{2}+3 a w+b\right) x^{2}+\left(4 w^{3}+3 a w^{2}+2 b w+c\right) x+w^{4}+a w^{3}+b w^{2}+c w+d$. Hence, $r_{g}(x)=g(x-(4 w+a) / 4)=g(x-w-a / 4)=f(x-w-a / 4+w)=f(x-a / 4)=r_{f}(x)$.

Finally, let $r_{f}(x)=r_{g}(x)$. Then $f(x-a / 4)=g(x-\bar{a} / 4)$. Hence, $f(x-a / 4+\bar{a} / 4)=g(x-$ $\bar{a} / 4+\bar{a} / 4)=g(x)$ and $g(x)=f(x-(\bar{a}-a) / 4)$ follows. Put $w=(\bar{a}-a) / 4$. Clearly, if $a \equiv \bar{a}$ $(\bmod 4)$, then $w \in \mathbb{Z}$. Suppose that $a \neq \equiv \bar{a}(\bmod 4)$. Using $(2.5)$ we obtain $R=8 b-3 a^{2}=8 \bar{b}-3 \bar{a}^{2}$, $S=8 c-4 a b+a^{3}=8 \bar{c}-4 \bar{a} \bar{b}+\bar{a}^{3}$, which implies $a^{2} \equiv \bar{a}^{2}(\bmod 8)$ and $a^{3} \equiv \bar{a}^{3}(\bmod 4)$. Therefore, $a^{2} \equiv \bar{a}^{2}(\bmod 4)$, which yields, without loss of generality, that either $a \equiv 0(\bmod 4), \bar{a} \equiv 2(\bmod 4)$ or $a \equiv 1(\bmod 4), \bar{a} \equiv 3(\bmod 4)$. If $a \equiv 0(\bmod 4), \bar{a} \equiv 2(\bmod 4)$, then $a^{2} \equiv 0(\bmod 8), \bar{a}^{2} \equiv 4$ $(\bmod 8)$, which is in contradiction to $a^{2} \equiv \bar{a}^{2}(\bmod 8)$. Similarly, if $a \equiv 1(\bmod 4), \bar{a} \equiv 3(\bmod 4)$, then $a^{3} \equiv 1(\bmod 4), \bar{a}^{3} \equiv 3(\bmod 4)$, which is in contradiction to $a^{3} \equiv \bar{a}^{3}(\bmod 4)$.

Let $0 \neq D \in \mathbb{Z}$ and let $Q_{D} \neq \emptyset$. For $f(x), g(x) \in Q_{D}$ put

$$
f(x) \sim g(x) \Longleftrightarrow \exists w \in \mathbb{Z}: g(x)=f(x+w)=f_{w}(x) \Longleftrightarrow r_{f}(x)=r_{g}(x) .
$$

It is evident that $\sim$ is an equivalence relation on the set $Q_{D}$. Moreover, $Q_{D} / \sim$ has only finitely many equivalence classes. In Section 4 , this fact will be proved using the results of Mordell and Siegel presented in Theorem 1.1 and Theorem 1.2. On the other hand, this claim also follows as a consequence of a more general theorem that has been proved by Kálmán Györy [8. p. 419]. See also [9. p. 475] or consult [3. p. 109].

\section{Connection between Mordell's equation $Y^{2}=X^{3}-2{ }^{16} 3^{3} D$ and the set $Q_{D}$}

Theorem 3.1. Let $0 \neq D \in \mathbb{Z}$. If Mordell's equation

$$
Y^{2}=X^{3}+k \quad \text { with } \quad k=-1769472 D=-2{ }^{16} 3^{3} D
$$

has no integer solution, then $Q_{D}=\emptyset$.

Pro of. Let $f(x)=x^{4}+a x^{3}+b x^{2}+c x+d \in Q_{D}$ and let $r_{f}(x)=x^{4}+A x^{2}+B x+C \in \mathbb{Q}[x]$. Direct calculation will verify that 2.3 can be written in the form

$$
D_{r_{f}}=\frac{4}{27}\left(A^{2}+12 C\right)^{3}-\frac{1}{27}\left(2 A^{3}-72 A C+27 B^{2}\right)^{2} .
$$

Substituting (2.4) into 3.2 , after short calculation, we obtain

$$
D_{r_{f}}=\frac{1}{1769472}\left(\left(R^{2}+3 T\right)^{3}-\left(R^{3}-9 R T+108 S^{2}\right)^{2}\right) .
$$

Put

$$
X=R^{2}+3 T \quad \text { and } \quad Y=R^{3}-9 R T+108 S^{2} .
$$

Then $X, Y \in \mathbb{Z}$ and $(3.3)$ yields

$$
Y^{2}=X^{3}+k \quad \text { where } \quad k=-1769472 D_{r_{f}}=-2{ }^{16} 3^{3} D_{r_{f}} .
$$

Since $D_{r_{f}}=D_{f}=D$, the proof is complete. 
Remark 3.2. If $x^{4}+a x^{3}+b x^{2}+c x+d \in Q_{D}$, then $(2.2)$ yields $A, B, C \in \mathbb{Z} \Longleftrightarrow 4 \mid a$. In this case, we can write 3.2 in the form $V^{2}=4 U^{3}-27 D_{r_{f}}$, where $U=A^{2}+12 C$ and $V=2 A^{3}-72 A C+27 B^{2}$. Hence, we have

$$
(4 V)^{2}=(4 U)^{3}-432 D_{r_{f}} .
$$

Since $D_{r_{f}}=D$, the substitutions $X=4 U, Y=4 V$ reduce 3.5 to

$$
Y^{2}=X^{3}-432 D \text {. }
$$

It is interesting that Mordell's equation (3.6) plays a fundamental role also in the theory of cubic polynomials with the same discriminant $D$. Consult $[10$ p. 313$]$.

The following notation will be useful. For an arbitrary $0 \neq D \in \mathbb{Z}$, let $M_{D}$ denote the set of all $\left[X_{0}, Y_{0}\right]$, where $X_{0}, Y_{0} \in \mathbb{Z}$ and $Y_{0}^{2}=X_{0}^{3}-2^{16} 3^{3} D$.

Lemma 3.3. Let $0 \neq D \in \mathbb{Z}$ and let $\left[X_{0}, Y_{0}\right] \in M_{D}$. Then (i), (ii), (iii) and (iv) hold:

(i) If $2 \mid X_{0}$, then $4\left|X_{0}, 8\right| Y_{0}$.

(ii) If $2 \mid Y_{0}$, then $4\left|X_{0}, 8\right| Y_{0}$.

(iii) If $3 \mid X_{0}$, then $9 \mid Y_{0}$.

(iv) If $3 \mid Y_{0}$, then $3\left|X_{0}, 9\right| Y_{0}$.

Pro of. The conclusions (i)-(iv) immediately follow from $Y_{0}^{2}=X_{0}^{3}-2^{16} 3^{3} D$.

They will be used in Section 4 and Section 5 .

\section{Method for determining the set $Q_{D}$}

The next lemma will be needed in the proof of Theorem 4.2

Lемма 4.1. Let $\xi_{0}, \eta_{0}, e \in \mathbb{Z}$ be such that

$$
\xi_{0} \equiv 36 e^{2} \quad(\bmod 96) \quad \text { and } \quad \eta_{0} \equiv 9 e \xi_{0}-108 e^{3}(\bmod 1728) .
$$

Then we have:

(i) $\xi_{0} \equiv 0(\bmod 12)$ and $\eta_{0} \equiv 0(\bmod 216)$.

(ii) There exists exactly one $e \in\{0,1,2,3\}$ satisfying (4.1).

Proof. (i) Since the validity of the congruence $\xi_{0} \equiv 0(\bmod 12)$ is evident, we only prove that $\eta_{0} \equiv 0(\bmod 216)$. First, observe that $\eta_{0} \equiv 9 e \xi_{0}-108 e^{3}(\bmod 216)$. Further, $\xi_{0} \equiv 36 e^{2}(\bmod 96)$ is equivalent to $9 \xi_{0} \equiv 324 e^{2}(\bmod 864)$. Hence, $9 \xi_{0} \equiv 108 e^{2}(\bmod 216)$. This, together with $\eta_{0} \equiv 9 e \xi_{0}-108 e^{3}(\bmod 216)$, yields $\eta_{0} \equiv 0(\bmod 216)$.

(ii) Let $\xi_{0}, \eta_{0} \in \mathbb{Z}$ satisfy 4.1 for some $e \in\{0,1,2,3\}$. Suppose that $e$ is not unique. Then it follows from $\xi_{0} \equiv 36 e^{2}(\bmod 96)$ that $e \in\{1,3\}$ and that $\xi_{0} \equiv 36(\bmod 96)$. On the other hand, using $\eta_{0} \equiv 9 e \xi_{0}-108 e^{3}(\bmod 1728)$, we obtain $9 \xi_{0}-108 \equiv 27 \xi_{0}-2916(\bmod 1728)$, which yields $\xi_{0} \equiv 60(\bmod 96)$, a contradiction.

The following Theorem 4.2 provides the necessary and sufficient condition for $Q_{D} \neq \emptyset$. In addition, Theorem 4.2 makes it possible to determine a particular polynomial in $Q_{D}$. 
Theorem 4.2. Let $0 \neq D \in \mathbb{Z}$ and let $M_{D} \neq \emptyset$. Then $Q_{D} \neq \emptyset$ if and only if there exists an $\left[X_{0}, Y_{0}\right] \in M_{D}$ such that the elliptic equation

$$
\eta^{2}=\xi^{3}-108 X_{0} \xi+432 Y_{0}
$$

has at least one integer solution $\left[\xi_{0}, \eta_{0}\right]$ satisfying conditions 4.3 4.5

$$
\begin{aligned}
36 e^{2}-\xi_{0} & \equiv 0(\bmod 96), \\
108 e^{3}-9 e \xi_{0}+\eta_{0} & \equiv 0(\bmod 1728), \\
432 e^{4}-\xi_{0}^{2}-72 e^{2} \xi_{0}+16 e \eta_{0}+144 X_{0} & \equiv 0(\bmod 110592) .
\end{aligned}
$$

for some $e \in\{0,1,2,3\}$. In this case,

$g(x)=x^{4}+e x^{3}+\frac{36 e^{2}-\xi_{0}}{96} x^{2}+\frac{108 e^{3}-9 e \xi_{0}+\eta_{0}}{1728} x+\frac{432 e^{4}-\xi_{0}^{2}-72 e^{2} \xi_{0}+16 e \eta_{0}+144 X_{0}}{110592} \in Q_{D}$ and

$$
r_{g}(x)=x^{4}-\frac{\xi_{0}}{96} x^{2}+\frac{\eta_{0}}{1728} x+\frac{144 X_{0}-\xi_{0}^{2}}{110592} .
$$

Proof. First, assume that $Q_{D} \neq \emptyset$. Then there exists an $f(x)=x^{4}+a x^{3}+b x^{2}+c x+d \in Q_{D}$ such that $r_{f}(x)=x^{4}+(R / 8) x^{2}+(S / 8) x+T / 256 \in \mathbb{Q}[x]$ where $R, S, T$ are integers satisfying $[2.5]$. Further, from Theorem 3.1 it follows that there exists a $\left[X_{0}, Y_{0}\right] \in M_{D}$ such that $R^{2}+3 T=X_{0}$ and $R^{3}-9 R T+108 S^{2}=Y_{0}$. Substituting $3 T=X_{0}-R^{2}$ into $R^{3}-9 R T+108 S^{2}=Y_{0}$, we obtain

$$
4 R^{3}-3 X_{0} R+108 S^{2}=Y_{0},
$$

and multiplying (4.6) by 432 , we get

$$
(216 S)^{2}=(-12 R)^{3}-108 X_{0}(-12 R)+432 Y_{0} .
$$

Put $\xi_{0}=-12 R$ and $\eta_{0}=216 S$. Now, 4.7 implies immediately that $\left[\xi_{0}, \eta_{0}\right]$ is an integer solution of 4.2 .

Finally, we have to prove that $\left[\xi_{0}, \eta_{0}\right]$ satisfies $4.3-4.5$ for some $e \in\{0,1,2,3\}$. Since $a \in \mathbb{Z}$, there exist uniquely determined $w \in \mathbb{Z}$ and $e \in\{0,1,2,3\}$ such that $a=4 w+e$. Substituting $a=4 w+e$ into the first equation of 2.5 , we obtain $R \equiv-3 e^{2}(\bmod 8)$ and $-12 R \equiv 36 e^{2}$ (mod 96) follows. This together with $\xi_{0}=-12 R$ yields $\xi_{0} \equiv 36 e^{2}(\bmod 96)$. Hence, (4.3).

Further, from the second equation of $(2.5)$, it follows

$$
216 S=1728 c-864 a b+216 a^{3} .
$$

Putting $a=4 w+e, 8 b=R+3 a^{2}, \xi_{0}=-12 R$ and $\eta_{0}=216 S$ into 4.8), we obtain

$$
\eta_{0}=1728\left(c-4 w^{3}-3 e w^{2}\right)+36 w\left(\xi_{0}-36 e^{2}\right)+9 e \xi_{0}-108 e^{3} .
$$

Reducing (4.9) by modulus 1728 and using $\xi_{0} \equiv 36 e^{2}(\bmod 96)$, we get (4.4).

Finally, the third equation of 2.5 implies

$$
432 T=110592 d-27648 a c+6912 a^{2} b-1296 a^{4} .
$$

For the left-hand side of 4.10$)$, we have $432 T=144\left(X_{0}-R^{2}\right)=144 X_{0}-\xi_{0}^{2}$ and the right-hand side of 4.10 can be rewritten, substituting $a=4 w+e, 8 b=R+3 a^{2}, 8 c=S+4 a b-a^{3}, \xi_{0}=-12 R$ and $\eta_{0}=216 S$ into

$$
110592\left(d-w^{4}-e w^{3}\right)-64 w\left(\eta_{0}-9 e \xi_{0}+108 e^{3}\right)-1152 w^{2}\left(36 e^{2}-\xi_{0}\right)-16 e \eta_{0}+72 e^{2} \xi_{0}-432 e^{4} .
$$

Since $\eta_{0} \equiv 9 e \xi_{0}-108 e^{3}(\bmod 1728)$ and $\xi_{0} \equiv 36 e^{2}(\bmod 96)$, we get

$$
144 X_{0}-\xi_{0}^{2} \equiv-16 e \eta_{0}+72 e^{2} \xi_{0}-432 e^{4}(\bmod 110592) .
$$

Hence, 4.5. 
Conversely, assume that there exists a $\left[X_{0}, Y_{0}\right] \in M_{D}$ such that equation 4.2 has an integer solution $\left[\xi_{0}, \eta_{0}\right]$ satisfying (4.3)-4.5) for some $e \in\{0,1,2,3\}$. Put

$$
R=\frac{-\xi_{0}}{12}, \quad S=\frac{\eta_{0}}{216}, \quad T=\frac{144 X_{0}-\xi_{0}^{2}}{432} .
$$

Then, by part (i) of Lemma 4.1, we have $R, S \in \mathbb{Z}$. We now prove that $T \in \mathbb{Z}$. From the first and third equation in 4.11 we obtain $T=\left(X_{0}-R^{2}\right) / 3$. First we show that

$$
X_{0} \equiv 0(\bmod 3) \Longleftrightarrow R \equiv 0(\bmod 3) .
$$

Let $3 \mid X_{0}$. Then, by part (iii) of Lemma 3.3 we have $9 \mid Y_{0}$. Further, by (4.11), we have $3 \mid \xi_{0}$ and $3^{3} \mid \eta_{0}$. Since $\eta_{0}^{2}=\xi_{0}^{3}-108 X_{0} \xi_{0}+432 Y_{0}$, we also have $0 \equiv \eta_{0}^{2} \equiv \xi_{0}^{3}\left(\bmod 3^{5}\right)$ and $\xi_{0} \equiv 0\left(\bmod 3^{2}\right)$ follows. This together with $\xi_{0}=-12 R$ yields $3 \mid R$.

Let $3 \mid R$. Since $\xi_{0}=-12 R$, we have $3^{2} \mid \xi_{0}$ and $3^{6} \mid \xi_{0}^{3}$ follows. Next, by (4.11), $3^{6} \mid \eta_{0}^{3}$. Since $\eta_{0}^{2}=\xi_{0}^{3}-108 X_{0} \xi_{0}+432 Y_{0}$, we have $432 Y_{0} \equiv 0\left(\bmod 3^{5}\right)$, and $Y_{0} \equiv 0\left(\bmod 3^{2}\right)$ follows. By part (iv) of Lemma 3.3 , we get $3 \mid X_{0}$. This proves 4.12 .

Further, suppose that $X_{0} \equiv 2(\bmod 3)$. Then from $\left[X_{0}, Y_{0}\right] \in M_{D}$ it follows that $Y_{0}^{2} \equiv 2$ (mod 3), which is a contradiction. Combining this fact with 4.12 , we get

$$
X_{0} \equiv 1(\bmod 3) \Longleftrightarrow R \equiv 1(\bmod 3) \quad \text { or } \quad R \equiv 2(\bmod 3) \Longleftrightarrow R^{2} \equiv 1(\bmod 3) .
$$

Clearly, in both cases 4.12$)$ and $(4.13)$, we have $X_{0}-R^{2} \equiv 0(\bmod 3)$. Hence, $T \in \mathbb{Z}$.

Consider now the polynomial

$$
r(x)=x^{4}+\frac{R}{8} x^{2}+\frac{S}{8} x+\frac{T}{256} \in \mathbb{Q}[x] .
$$

We prove that the discriminant $D_{r}$ of $r(x)$ is equal to $D$. First, direct calculation verifies that

$$
D_{r}=\frac{\left(R^{2}+3 T\right)^{3}-\left(R^{3}-9 R T+108 S^{2}\right)^{2}}{2^{16} 3^{3}} .
$$

On the other hand, substituting $\xi_{0}=-12 R, \eta_{0}=216 S$ into $\eta_{0}^{2}=\xi_{0}^{3}-108 X_{0} \xi_{0}+432 Y_{0}$, we obtain

$$
(216 S)^{2}=(-12 R)^{3}-108 X_{0}(-12 R)+432 Y_{0} .
$$

Hence, we get $R^{3}-3 R\left(X_{0}-R^{2}\right)+108 S^{2}=Y_{0}$. Since, $X_{0}-R^{2}=3 T$, we have $R^{3}-9 R T+108 S^{2}=Y_{0}$. This, together with $Y_{0}^{2}=X_{0}^{3}-2^{16} 3^{3} D$ yields

$$
D=\frac{\left(R^{2}+3 T\right)^{3}-\left(R^{3}-9 R T+108 S^{2}\right)^{2}}{2^{16} 3^{3}} .
$$

Hence, $D_{r}=D$.

Finally, let $e \in\{0,1,2,3\}$ satisfy 4.3 -4.5. Then, by part (ii) of Lemma 4.1 $e$ is uniquely determined. Put $g(x)=r(x+e / 4)$. Then we obtain after some calculation that

$$
\begin{aligned}
g(x) & =x^{4}+e x^{3}+\frac{R+3 e^{2}}{8} x^{2}+\frac{e R+2 S+e^{3}}{16} x+\frac{2 e^{2} R+8 e S+T+e^{4}}{256} \\
& =x^{4}+e x^{3}+\frac{36 e^{2}-\xi_{0}}{96} x^{2}+\frac{108 e^{3}-9 e \xi_{0}+\eta_{0}}{1728} x+\frac{432 e^{4}-\xi_{0}^{2}-72 e^{2} \xi_{0}+16 e \eta_{0}+144 X_{0}}{110592}
\end{aligned}
$$

and

$$
r_{g}(x)=r(x)=x^{4}-\frac{\xi_{0}}{96} x^{2}+\frac{\eta_{0}}{1728} x+\frac{144 X_{0}-\xi_{0}^{2}}{110592} .
$$

Since $D_{g}=D_{r_{g}}=D_{r}=D$, we have $g(x) \in Q_{D}$, as desired. The proof is complete. 
JIŘÍ KLAŠKA

Before proceeding, the following notations will be adopted. For any $\left[X_{0}, Y_{0}\right] \in M_{D}$, let $E_{D}\left(X_{0}, Y_{0}\right)$ denote the set of all $\left[\xi_{0}, \eta_{0}\right]$ where $\xi_{0}, \eta_{0} \in \mathbb{Z}$ and $\eta_{0}^{2}=\xi_{0}^{3}-108 X_{0} \xi_{0}+432 Y_{0}$. Next, let $E_{D}$ denote the set of all $\left[X_{0}, Y_{0}, \xi_{0}, \eta_{0}, e\right]$ where $\left[X_{0}, Y_{0}\right] \in M_{D},\left[\xi_{0}, \eta_{0}\right] \in E_{D}\left(X_{0}, Y_{0}\right)$ and $e \in\{0,1,2,3\}$ satisfy $4.3-4.5$.

Corollary 4.3. Let $0 \neq D \in \mathbb{Z}$ and let $f(x)=x^{4}+a x^{3}+b x^{2}+c x+d \in \mathbb{Z}[x]$. Then $f(x) \in Q_{D}$ if and only if there exists $\left[X_{0}, Y_{0}, \xi_{0}, \eta_{0}, e\right] \in E_{D}$ and $w \in \mathbb{Z}$ such that

$$
\begin{aligned}
& a=4 w+e, \\
& b=6 w^{2}+3 e w+\frac{36 e^{2}-\xi_{0}}{96}, \\
& c=4 w^{3}+3 e w^{2}+\frac{36 e^{2}-\xi_{0}}{48} w+\frac{108 e^{3}-9 e \xi_{0}+\eta_{0}}{1728}, \\
& d=w^{4}+e w^{3}+\frac{36 e^{2}-\xi_{0}}{96} w^{2}+\frac{108 e^{3}-9 e \xi_{0}+\eta_{0}}{1728} w+\frac{432 e^{4}-\xi_{0}^{2}-72 e^{2} \xi_{0}+16 e \eta_{0}+144 X_{0}}{110592} .
\end{aligned}
$$

Proposition 4.4. Let $0 \neq D \in \mathbb{Z}$ and let $M_{D} \neq \emptyset$. Then (i), (ii) and (iii) hold:

(i) $E_{D}\left(X_{0}, Y_{0}\right)$ is a finite set for any $\left[X_{0}, Y_{0}\right] \in M_{D}$.

(ii) $E_{D}$ is a finite set.

(iii) $Q_{D} / \sim$ has only finitely many equivalence classes for any $Q_{D} \neq \emptyset$.

Pro of. (i) Put $\alpha=-108 X_{0}$ and $\beta=432 Y_{0}$. Then $4 \alpha^{3}+27 \beta^{2}=2^{8} 3^{9}\left(-X_{0}^{3}+Y_{0}^{2}\right)=-2^{24} 3^{12} D \neq 0$. Conclusion (i) now follows from Theorem 1.2 .

(ii) Conclusion (ii) is a direct consequence of Theorem 1.1 and part (i) of Proposition 4.4

(iii) Let $\varphi: E_{D} \rightarrow Q_{D} / \sim$ be the mapping defined by $\varphi\left(X_{0}, Y_{0}, \xi_{0}, \eta_{0}, e\right)=\left\{f_{w}(x) ; w \in \mathbb{Z}\right\}$, where

$$
f_{0}(x)=x^{4}+e x^{3}+\frac{36 e^{2}-\xi_{0}}{96} x^{2}+\frac{108 e^{3}-9 e \xi_{0}+\eta_{0}}{1728} x+\frac{432 e^{4}-\xi_{0}^{2}-72 e^{2} \xi_{0}+16 e \eta_{0}+144 X_{0}}{110592} .
$$

Then $\varphi$ is bijective. Injectivity of $\varphi$ is evident and surjectivity of $\varphi$ immediately follows from Corollary 4.3. Hence, $\# Q_{D} / \sim=\# E_{D}$. This proves (iii).

Remark 4.5. Let $\left[X_{0}, Y_{0}\right],\left[X_{0}^{*}, Y_{0}^{*}\right] \in M_{D}$ and let $\left[X_{0}, Y_{0}\right] \neq\left[X_{0}^{*}, Y_{0}^{*}\right]$. By an example we will prove that the set $E_{D}\left(X_{0}, Y_{0}\right) \cap E_{D}\left(X_{0}^{*}, Y_{0}^{*}\right)$ can be nonempty. For $D=-23$, we have $[64,6400],[-320,-2816] \in M_{-23}$ and $[96, \pm 1728] \in E_{-23}(64,6400) \cap E_{-23}(-320,-2816)$.

Now we are ready to formulate the method for determining the set $Q_{D}$. It can be formally divided into five steps as follows:

(i) Let $0 \neq D \in \mathbb{Z}$. First we find the set $M_{D}$ of all integer solutions $\left[X_{0}, Y_{0}\right]$ of Mordell's equation $Y^{2}=X^{3}-2^{16} 3^{3} D$. By Theorem 1.1, $M_{D}$ is a finite set and Theorem 3.1 states that, if $M_{D}=\emptyset$, then $Q_{D}=\emptyset$.

(ii) Let $M_{D} \neq \emptyset$. Next we find, for any $\left[X_{0}, Y_{0}\right] \in M_{D}$, the set $E_{D}\left(X_{0}, Y_{0}\right)$ of all integer solutions $\left[\xi_{0}, \eta_{0}\right]$ of the elliptic equation $\eta^{2}=\xi^{3}-108 X_{0} \xi+432 Y_{0}$. By part (i) of Proposition $4.4, E_{D}\left(X_{0}, Y_{0}\right)$ is a finite set for any $\left[X_{0}, Y_{0}\right] \in M_{D}$ and Theorem 4.2 says that, if $E_{D}\left(X_{0}, Y_{0}\right)=\emptyset$ for any $\left[X_{0}, Y_{0}\right] \in M_{D}$, then $Q_{D}=\emptyset$.

(iii) In step (iii), we establish the set $E_{D}$. By part (ii) of Proposition 4.4, $E_{D}$ is a finite set and Corollary 4.3 states that $Q_{D} \neq \emptyset$ if and only if $E_{D} \neq \emptyset$.

(iv) Let $E_{D} \neq \emptyset$ and let $\# E_{D}=n$. In this step, we assign to each $\left[X_{0}, Y_{0}, \xi_{0}, \eta_{0}, e\right] \in E_{D}$ the polynomial

$$
g(x)=x^{4}+e x^{3}+\frac{36 e^{2}-\xi_{0}}{96} x^{2}+\frac{108 e^{3}-9 e \xi_{0}+\eta_{0}}{1728} x+\frac{432 e^{4}-\xi_{0}^{2}-72 e^{2} \xi_{0}+16 e \eta_{0}+144 X_{0}}{110592} .
$$




\section{QUARTIC POLYNOMIALS WITH A GIVEN DISCRIMINANT}

In this way, we obtain the full system of representatives $G_{D}=\left\{g_{1}(x), \ldots, g_{n}(x)\right\}$ of $Q_{D} / \sim$. By part (iii) of Proposition 4.4. $Q_{D} / \sim$ is a finite set.

(v) Finally, applying Corollary 2.3 to each $g_{i}(x) \in G_{D}, i \in\{1, \ldots, n\}$, we obtain the $n$ sets $\left\{f_{i, w}(x) ; w \in \mathbb{Z}\right\}$ where

$$
f_{i, w}(x)=x^{4}+\frac{g_{i}^{\prime \prime \prime}(w)}{3 !} x^{3}+\frac{g_{i}^{\prime \prime}(w)}{2 !} x^{2}+\frac{g_{i}^{\prime}(w)}{1 !} x+g_{i}(w) .
$$

Hence, we get

$$
Q_{D}=\bigcup_{i=1}^{n}\left\{f_{i, w}(x) ; w \in \mathbb{Z}\right\} .
$$

The below example illustrates our method.

Example 4.6. Let $D=-87$. Then we have

$$
M_{-87}=\{[-320, \pm 11008],[-92, \pm 12376],[448, \pm 15616]\} .
$$

Hence,

$$
\begin{aligned}
& E_{-87}(-320,11008)=\{[-80, \pm 1216],[-48, \pm 1728],[240, \pm 5184],[384, \pm 8640],[8592, \pm 796608]\}, \\
& E_{-87}(-320,-11008)=\emptyset, \\
& E_{-87}(-92,12376)=\{[-156,0]\}, \\
& E_{-87}(-92,-12376)=\{[156,0]\}, \\
& E_{-87}(448,15616)=\{[-156, \pm 3240],[96, \pm 1728]\}, \\
& E_{-87}(448,-15616)=\emptyset .
\end{aligned}
$$

Further, we have

$$
\begin{aligned}
E_{-87}=\{ & {[-320,11008,240,5184,2],[-320,11008,240,-5184,2], } \\
& {[448,15616,-156,-3240,1],[448,15616,-156,3240,3]\} . }
\end{aligned}
$$

Hence, it follows that $\# E_{-87}=\# Q_{-87} / \sim=4$ and that $G_{-87}=\left\{g_{1}(x), g_{2}(x), g_{3}(x), g_{4}(x)\right\}$ where

$$
\begin{array}{ll}
g_{1}(x)=x^{4}+2 x^{3}-x^{2}+x, & g_{2}(x)=x^{4}+2 x^{3}-x^{2}-5 x-3, \\
g_{3}(x)=x^{4}+x^{3}+2 x^{2}-x, & g_{4}(x)=x^{4}+3 x^{3}+5 x^{2}+6 x+3 .
\end{array}
$$

Finally,

$$
\begin{aligned}
& f_{1, w}(x)=x^{4}+(4 w+2) x^{3}+\left(6 w^{2}+6 w-1\right) x^{2}+\left(4 w^{3}+6 w^{2}-2 w+1\right) x+w^{4}+2 w^{3}-w^{2}+w, \\
& f_{2, w}(x)=x^{4}+(4 w+2) x^{3}+\left(6 w^{2}+6 w-1\right) x^{2}+\left(4 w^{3}+6 w^{2}-2 w-5\right) x+w^{4}+2 w^{3}-w^{2}-5 w-3, \\
& f_{3, w}(x)=x^{4}+(4 w+1) x^{3}+\left(6 w^{2}+3 w+2\right) x^{2}+\left(4 w^{3}+3 w^{2}+4 w-1\right) x+w^{4}+w^{3}+2 w^{2}-w, \\
& f_{4, w}(x)=x^{4}+(4 w+3) x^{3}+\left(6 w^{2}+9 w+5\right) x^{2}+\left(4 w^{3}+9 w^{2}+10 w+6\right) x+w^{4}+3 w^{3}+5 w^{2}+6 w+3,
\end{aligned}
$$

and

$$
Q_{-87}=\bigcup_{i=1}^{4}\left\{f_{i, w}(x) ; w \in \mathbb{Z}\right\} .
$$

Applying the method, the validity of Theorem 4.7 can be verified. 
TheOREM 4.7. Let $0 \neq D \in \mathbb{Z}$ and let $1 \leq|D| \leq 1000$. Then we have:

(i) If $1 \leq D \leq 1000$, then $Q_{D} \neq \emptyset$ if and only if $D \in\{5,12,20,21,32,40,45,48,49,60,77,81,85,96,104,112,117,125,140,144,148,165$ $169,189,192,216,221,224,229,252,256,257,260,272,285,288,320,321,333,357$, $361,392,400,404,432,437,468,469,473,480,488,500,512,525,528,533,544,549$, $564,572,580,592,605,621,629,656,672,697,725,729,733,761,768,785,788,792$, $816,832,837,864,892,896,900,916,957,981,985\}$.

(ii) If $-1 \geq D \geq-1000$, then $Q_{D} \neq \emptyset$ if and only if

$$
\begin{aligned}
D \in\{ & -3,-16,-23,-27,-31,-44,-59,-76,-83,-87,-107,-108,-112,-135,-139, \\
& -140,-175,-176,-199,-211,-231,-236,-240,-247,-255,-256,-268,-275, \\
& -279,-283,-288,-304,-331,-332,-335,-351,-367,-400,-416,-428,-432, \\
& -448,-464,-475,-491,-500,-507,-527,-556,-560,-563,-575,-588,-608, \\
& -643,-671,-684,-688,-695,-731,-751,-783,-800,-816,-844,-848,-863, \\
& -864,-891,-931,-944,-959,-972,-976,-983\} .
\end{aligned}
$$

\section{Structure of the set $Q_{D}$}

In this section, we establish some results related to the structure of the set $Q_{D}$. For $e \in$ $\{0,1,2,3\}$, put $Q_{D}(e)=\left\{x^{4}+a x^{3}+b x^{2}+c x+d \in Q_{D} ; a \equiv e(\bmod 4)\right\}$. Then $Q_{D}(0), Q_{D}(1)$, $Q_{D}(2), Q_{D}(3)$ are pairwise disjoint sets, and

$$
Q_{D}=\bigcup_{e=0}^{4} Q_{D}(e) .
$$

Proposition 5.1 gives the important link between the sets $Q_{D}(1)$ and $Q_{D}(3)$.

Proposition 5.1. Let $0 \neq D \in \mathbb{Z}$ and let $e \in\{1,3\}$. Then there exists a one-to-one correspondence between the sets $Q_{D}(1)$ and $Q_{D}(3)$ given by the relation

$$
\left[X_{0}, Y_{0}, \xi_{0}, \eta_{0}, 1\right] \in E_{D} \Longleftrightarrow\left[X_{0}, Y_{0}, \xi_{0},-\eta_{0}, 3\right] \in E_{D} .
$$

Consequently, $Q_{D}(1) \neq \emptyset$ if and only if $Q_{D}(3) \neq \emptyset$.

Proof. First observe that $\left[\xi_{0}, \eta_{0}\right] \in E_{D}\left(X_{0}, Y_{0}\right)$ if and only if $\left[\xi_{0},-\eta_{0}\right] \in E_{D}\left(X_{0}, Y_{0}\right)$.

If $e \in\{1,3\}$, then, by $(4.3), \xi_{0} \equiv 36(\bmod 96)$, which is equivalent to

$$
18 \xi_{0} \equiv 648(\bmod 1728) \text {. }
$$

Next, if $e=1$, then, by 4.4$), 9 \xi_{0}-\eta_{0}-108 \equiv 0(\bmod 1728)$. Using (5.1), this congruence can be written in the equivalent form

$$
1719 \xi_{0}+\eta_{0}+108 \equiv 27 \xi_{0}+94 \cdot 18 \xi_{0}+\eta_{0}+108 \equiv 27 \xi_{0}+\eta_{0}+540 \equiv 0 \quad(\bmod 1728) .
$$

Hence, $\left[\xi_{0}, \eta_{0}\right]$ satisfies (4.4) for $e=1$ if and only if $\left[\xi_{0},-\eta_{0}\right]$ satisfies (4.4) for $e=3$.

Furthermore, if $e \in\{1,3\}$, then, by 4.3$), \xi_{0} \equiv 36(\bmod 96)$, which is equivalent to

$$
1152 \xi_{0}-41472 \equiv 0 \quad(\bmod 110592) .
$$

Next, if $e=1$ then, from $(4.4)$, it follows that $\eta_{0} \equiv 9 \xi_{0}-108(\bmod 1728)$ if and only if

$$
576 \xi_{0}-64 \eta_{0}-6912 \equiv 0 \quad(\bmod 110592) .
$$


Subtracting (5.3) from (5.2), we now obtain

$$
576 \xi_{0}+64 \eta_{0}-34560 \equiv 0(\bmod 110592) .
$$

Finally, if $e=1$, then 4.5 yields

$$
432-\xi_{0}^{2}-72 \xi_{0}+16 \eta_{0}+144 X_{0} \equiv 0(\bmod 110592) .
$$

Subtracting (5.4) from (5.5), we obtain

$$
34992-\xi_{0}^{2}-648 \xi_{0}-48 \eta_{0}+144 X_{0} \equiv 0(\bmod 110592) .
$$

This proves that $\left[X_{0}, Y_{0}, \xi_{0}, \eta_{0}, 1\right] \in E_{D}$ if and only if $\left[X_{0}, Y_{0}, \xi_{0},-\eta_{0}, 3\right] \in E_{D}$, which implies $Q_{D}(1) \neq \emptyset$ if and only if $Q_{D}(3) \neq \emptyset$.

For the remaining cases $e \in\{0,2\}$, we can prove Proposition 5.2 .

Proposition 5.2. Let $0 \neq D \in \mathbb{Z}$ and let $e \in\{0,2\}$. Then we have:

(i) $\left[X_{0}, Y_{0}, \xi_{0}, \eta_{0}, 0\right] \in E_{D} \Longleftrightarrow\left[X_{0}, Y_{0}, \xi_{0},-\eta_{0}, 0\right] \in E_{D}$.

(ii) $\left[X_{0}, Y_{0}, \xi_{0}, \eta_{0}, 2\right] \in E_{D} \Longleftrightarrow\left[X_{0}, Y_{0}, \xi_{0},-\eta_{0}, 2\right] \in E_{D}$.

Proof. Since part (i) of Proposition 5.2 immediately follows from 4.3 - 4.5, we prove (ii). Let $\left[X_{0}, Y_{0}, \xi_{0}, \eta_{0}, 2\right] \in E_{D}$. Then 4.3$)-4.5$ yields that $\xi_{0} \equiv 48(\bmod 96), \eta_{0} \equiv 18 \xi_{0}-864$ $(\bmod 1728)$, and

$$
6912-\xi_{0}^{2}-288 \xi_{0}+32 \eta_{0}+144 X_{0} \equiv 0(\bmod 110592) .
$$

Since $\xi_{0} \equiv 48(\bmod 96)$ is equivalent to $18 \xi_{0} \equiv 864(\bmod 1728)$, we have $-\eta_{0} \equiv-18 \xi_{0}+864 \equiv$ $1710 \xi_{0}-864=18 \xi_{0}-864+94 \cdot 18 \xi_{0} \equiv 18 \xi_{0}-864+47 \cdot 1728 \equiv 18 \xi_{0}-864(\bmod 1728)$. Further, $\xi_{0} \equiv 48(\bmod 96)$ is equivalent to $1152 \xi_{0} \equiv 55296(\bmod 110592)$ and $\eta_{0} \equiv 18 \xi_{0}-864(\bmod 1728)$ is equivalent to $64 \eta_{0} \equiv 1152 \xi_{0}-55296(\bmod 110592)$. Hence, we obtain $64 \eta_{0} \equiv 0(\bmod 110592)$ and $32 \eta_{0} \equiv-32 \eta_{0}(\bmod 110592)$ follows. Now we see that (5.6) is equivalent to $6912-\xi_{0}^{2}-288 \xi_{0}-$ $32 \eta_{0}+144 X_{0} \equiv 0(\bmod 110592)$. This proves (ii).

Remark 5.3. If $Q_{D} \neq \emptyset$, then any of the below seven cases can occur:

(i) $Q_{D}(0) \neq \emptyset, Q_{D}(1) \cup Q_{D}(3) \neq \emptyset, Q_{D}(2) \neq \emptyset, D=-23$,

(ii) $Q_{D}(0) \neq \emptyset, Q_{D}(1) \cup Q_{D}(3) \neq \emptyset, Q_{D}(2)=\emptyset, D=32$,

(iii) $Q_{D}(0) \neq \emptyset, Q_{D}(1) \cup Q_{D}(3)=\emptyset, Q_{D}(2) \neq \emptyset, D=5$,

(iv) $Q_{D}(0)=\emptyset, Q_{D}(1) \cup Q_{D}(3) \neq \emptyset, Q_{D}(2) \neq \emptyset, D=-87$,

(v) $Q_{D}(0) \neq \emptyset, Q_{D}(1) \cup Q_{D}(3)=\emptyset, Q_{D}(2)=\emptyset, D=-27$,

(vi) $Q_{D}(0)=\emptyset, Q_{D}(1) \cup Q_{D}(3) \neq \emptyset, Q_{D}(2)=\emptyset, D=12$,

(vii) $Q_{D}(0)=\emptyset, Q_{D}(1) \cup Q_{D}(3)=\emptyset, Q_{D}(2) \neq \emptyset, D=-3$.

The above values of $D$ are the least, in absolute value, for which the case occurs.

\section{Even and odd solutions of Mordell's equation $Y^{2}=X^{3}-2^{16} 3^{3} D$}

Some basic arithmetic properties of integer solutions $\left[X_{0}, Y_{0}\right]$ of the Mordell's equation $Y^{2}=$ $X^{3}-2^{16} 3^{3} D$ have already been presented in Lemma 3.3 Combining part (i) and (ii) of Lemma 3.3 . we immediately get $X_{0} \equiv 0(\bmod 2) \Longleftrightarrow Y_{0} \equiv 0(\bmod 2)$. Hence, the following two definitions are possible:

(i) A solution $\left[X_{0}, Y_{0}\right] \in M_{D}$ is called even, if $X_{0}$ and $Y_{0}$ are even.

(ii) A solution $\left[X_{0}, Y_{0}\right] \in M_{D}$ is called odd, if $X_{0}$ and $Y_{0}$ are odd. 
Next, for any $0 \neq D \in \mathbb{Z}$, let

$$
\begin{aligned}
\mathcal{E}_{D} & =\left\{\left[X_{0}, Y_{0}\right] \in M_{D}: X_{0} \equiv Y_{0} \equiv 0(\bmod 2)\right\}, \\
\mathcal{O}_{D} & =\left\{\left[X_{0}, Y_{0}\right] \in M_{D}: X_{0} \equiv Y_{0} \equiv 1(\bmod 2)\right\} .
\end{aligned}
$$

Then $\mathcal{E}_{D} \cap \mathcal{O}_{D}=\emptyset$ and $\mathcal{E}_{D} \cup \mathcal{O}_{D}=M_{D}$. Finally, for any positive integer $n$, put

$$
\varepsilon_{n}=\sum_{D=1}^{n} \# \mathcal{E}_{D}, \quad \varepsilon_{-n}=\sum_{D=-1}^{-n} \# \mathcal{E}_{D}, \quad o_{n}=\sum_{D=1}^{n} \# \mathcal{O}_{D}, \quad \text { and } \quad o_{-n}=\sum_{D=-1}^{-n} \# \mathcal{O}_{D} .
$$

Computer investigation of the values $\varepsilon_{n}, \varepsilon_{-n}, o_{n}$ and $o_{-n}$ for $n \leq 1000$ reveals a significant difference between the numbers of even and odd solutions in the investigated range. We have found

$$
\varepsilon_{-1000}=1572, \quad \varepsilon_{1000}=1090, \quad o_{-1000}=100, \quad \text { and } \quad o_{1000}=44 .
$$

Hence, it follows that there exist approximately $95 \%$ even and only $5 \%$ odd integer solutions of $Y^{2}=X^{3}-2{ }^{16} 3^{3} D$ for $0 \neq|D| \leq 1000$. This surprising fact inspires the study of even solutions in detail. As the main result of this section, we prove that, for any even solution $\left[X_{0}, Y_{0}\right]$, equation (4.2) can be replaced by another elliptic equation whose integer coefficients are substantially smaller in the absolute value than in 4.2 .

We begin by recalling the well-known proposition concerning the solubility of linear congruences. See, for example, Hardy and Wright [4 p. 62, Theorem 57].

Proposition 6.1. Let $a, b, m \in \mathbb{Z}, m>1$ and let $g=\operatorname{gcd}(a, m)$. Then the congruence $a x \equiv b$ $(\bmod m)$ is soluble if and only if $g \mid b$.

Using Proposition 6.1 we now prove Lemma 6.2

Lemma 6.2. If $X_{0}, Y_{0} \in \mathbb{Z}$, then the congruence $3 \alpha X_{0}+Y_{0}-4 \alpha^{3} \equiv 0(\bmod 27)$ holds for at most one $\alpha \in\{0,1,2\}$.

Proof. The proof consists of three steps. (i) First, suppose that $Y_{0} \equiv 0(\bmod 27)$ and $3 X_{0}+$ $Y_{0}-4 \equiv 0(\bmod 27)$. Then $Y_{0} \equiv 4-3 X_{0} \equiv 0(\bmod 27)$, which yields $3 X_{0} \equiv 4(\bmod 27)$. Hence, $\operatorname{gcd}(3,27)=3 \nmid 4$, which is a contradiction.

(ii) Further, suppose that $Y_{0} \equiv 0(\bmod 27)$ and $6 X_{0}+Y_{0}-32 \equiv 0(\bmod 27)$. Then $Y_{0} \equiv$ $5-6 X_{0} \equiv 0(\bmod 27)$, which yields $6 X_{0} \equiv 5(\bmod 27)$. Hence, $\operatorname{gcd}(6,27)=3 \nmid 5$, which is a contradiction.

(iii) Finally, suppose that $3 X_{0}+Y_{0}-4 \equiv 0(\bmod 27)$ and $6 X_{0}+Y_{0}-32 \equiv 0(\bmod 27)$. Then $Y_{0} \equiv 4-3 X_{0} \equiv 5-6 X_{0}(\bmod 27)$, which yields $3 X_{0} \equiv 1(\bmod 27)$. Hence, $\operatorname{gcd}(3,27)=3 \nmid 1$, which is a contradiction. Combining (i)-(iii) proves the lemma.

We are now ready to prove the main result of this section.

TheоRem 6.3. Let $0 \neq D \in \mathbb{Z}$ and let $\left[X_{0}, Y_{0}\right] \in \mathcal{E}_{D}$.

(i) If $3 \alpha X_{0}+Y_{0}-4 \alpha^{3} \equiv 0(\bmod 27)$ does not hold for any $\alpha \in\{0,1,2\}$, then the system

$$
R^{2}+3 T=X_{0} \quad \text { and } \quad R^{3}-9 R T+108 S^{2}=Y_{0}
$$

is not solvable in integers.

(ii) If $3 \alpha X_{0}+Y_{0}-4 \alpha^{3} \equiv 0(\bmod 27)$ holds for some $\alpha \in\{0,1,2\}$, then $\alpha$ is uniquely determined, $X_{0}-4 \alpha^{2} \equiv 0(\bmod 12), 3 \alpha X_{0}+Y_{0}-4 \alpha^{3} \equiv 0 \quad(\bmod 108)$ and the set $K$ of all integer solutions of (6.1) can be obtained from the set $L$ of all integer solutions of the elliptic equation

$$
\eta^{2}=\xi^{3}-\alpha \xi^{2}-\frac{X_{0}-4 \alpha^{2}}{12} \xi+\frac{3 \alpha X_{0}+Y_{0}-4 \alpha^{3}}{108} .
$$

Moreover, between $K$ and $L$, there exists a one-to-one correspondence. 
Proof. (i) Let $\left[R_{0}, S_{0}, T_{0}\right]$ be an arbitrary integer solution of (6.1). Since $R_{0} \in \mathbb{Z}$, there exits a uniquely determined $r \in \mathbb{Z}$ and $\alpha \in\{0,1,2\}$ such that $R_{0}=3 r+\alpha$.

First, we prove

$$
\frac{X_{0}-4 \alpha^{2}}{12} \in \mathbb{Z} \Longleftrightarrow 2 \mid X_{0}
$$

Let $2 \mid X_{0}$. Then, by part (i) of Lemma 3.3. $4 \mid X_{0}$, and thus $4 \mid X_{0}-4 \alpha^{2}$. Next, the first equation in (6.1) yields $X_{0}-R_{0}^{2} \equiv X_{0}-\alpha^{2} \equiv 0(\bmod 3)$, and $3 \mid X_{0}-4 \alpha^{2}$ follows. Hence, $12 \mid X_{0}-4 \alpha^{2}$, which means $\left(X_{0}-4 \alpha^{2}\right) / 12 \in \mathbb{Z}$. Because the validity of the converse implication is evident, we get 6.3).

Further, substituting $3 T_{0}=X_{0}-R_{0}^{2}$ into $R_{0}^{3}-9 R_{0} T_{0}+108 S_{0}^{2}=Y_{0}$, we obtain

$$
4 R_{0}^{3}-3 X_{0} R_{0}+108 S_{0}^{2}=Y_{0} .
$$

Since $R_{0}=3 r+\alpha, 6.4$ can be written in the equivalent form

$$
108 S_{0}^{2}=-108 r^{3}-108 \alpha r^{2}+9\left(X_{0}-4 \alpha^{2}\right) r+3 \alpha X_{0}+Y_{0}-4 \alpha^{3} .
$$

Reducing (6.5) by the modulus 108 , using 6.3$)$, we get $3 \alpha X_{0}+Y_{0}-4 \alpha^{3} \equiv 0(\bmod 108)$. This proves (i).

(ii) Assume that there exists an $\alpha \in\{0,1,2\}$ such that $3 \alpha X_{0}+Y_{0}-4 \alpha^{3} \equiv 0(\bmod 27)$. Then Lemma 6.2 states that $\alpha$ is uniquely determined. Since $\left[X_{0}, Y_{0}\right] \in \mathcal{E}_{D}$, by part (i) of Lemma 3.3 . $4 \mid X_{0}$ and $8 \mid Y_{0}$, which yields $4 \mid 3 \alpha X_{0}+Y_{0}-4 \alpha^{3}$ for any $\alpha \in\{0,1,2\}$. Hence,

$$
3 \alpha X_{0}+Y_{0}-4 \alpha^{3} \equiv 0(\bmod 27) \Longleftrightarrow 3 \alpha X_{0}+Y_{0}-4 \alpha^{3} \equiv 0(\bmod 108) .
$$

We now prove that

$$
3 \alpha X_{0}+Y_{0}-4 \alpha^{3} \equiv 0(\bmod 27) \Longrightarrow X_{0}-4 \alpha^{2} \equiv 0(\bmod 12) .
$$

First, by part (i) of Lemma 3.3, $X_{0}-4 \alpha^{2} \equiv 0(\bmod 4)$. Next, from the assumption $3 \alpha X_{0}+$ $Y_{0}-4 \alpha^{3} \equiv 0(\bmod 27)$, we get $Y_{0} \equiv \alpha^{3} \equiv \alpha(\bmod 3)$. Hence, $Y_{0}^{2} \equiv \alpha^{2}(\bmod 3)$. Furthermore, it is clear from $\left[X_{0}, Y_{0}\right] \in \mathcal{E}_{D}$ that $Y_{0}^{2} \equiv X_{0}^{3}(\bmod 3)$, which, together with $X_{0}^{3} \equiv X_{0}(\bmod 3)$, yields $X_{0} \equiv \alpha^{2}(\bmod 3)$. Hence, $X_{0}-4 \alpha^{2} \equiv 0(\bmod 3)$, and 6.6$)$ follows.

Let $\left[R_{0}, S_{0}, T_{0}\right] \in K$. Then there exist a uniquely determined $r \in \mathbb{Z}$ and an $\alpha \in\{0,1,2\}$ such that $R_{0}=3 r+\alpha$. In much the same way as in the proof of part (i), we get (6.5). Hence,

$$
S_{0}^{2}=-r^{3}-\alpha r^{2}+\frac{X_{0}-4 \alpha^{2}}{12} r+\frac{3 \alpha X_{0}+Y_{0}-4 \alpha^{3}}{108}
$$

where $\left(X_{0}-4 \alpha^{2}\right) / 12$ and $\left(3 \alpha X_{0}+Y_{0}-4 \alpha^{3}\right) / 108$ are integers. Put $\left[\xi_{0}, \eta_{0}\right]=\left[-r, S_{0}\right]$. Then it follows from (6.7) that $\left[\xi_{0}, \eta_{0}\right] \in L$.

Conversely, let $\left[\xi_{0}, \eta_{0}\right] \in L$ where $\alpha \in\{0,1,2\}$ satisfies $3 \alpha X_{0}+Y_{0}-4 \alpha^{3} \equiv 0(\bmod 27)$. Put $R_{0}=-3 \xi_{0}+\alpha$ and $S_{0}=\eta_{0}$. Then $R_{0}, S_{0} \in \mathbb{Z}$, and substituting $\xi_{0}=\left(\alpha-R_{0}\right) / 3, \eta_{0}=S_{0}$ into (6.2) some calculation will yield

$$
R_{0}^{3}+3 R_{0}\left(R_{0}^{2}-X_{0}\right)+108 S_{0}^{2}=Y_{0} .
$$

Next, put $T_{0}=\left(X_{0}-R_{0}^{2}\right) / 3$. Since $R_{0}=-3 \xi_{0}+\alpha$, we have $T_{0}=2 \alpha \xi_{0}-3 \xi_{0}^{2}+\left(X_{0}-\alpha^{2}\right) / 3$, and by (6.6), $T_{0} \in \mathbb{Z}$. Substituting $R_{0}^{2}-X_{0}=-3 T_{0}$ into (6.8), we obtain $R_{0}^{3}-9 R_{0} T_{0}+108 S_{0}^{2}=Y_{0}$. This proves that $\left[R_{0}, S_{0}, T_{0}\right]=\left[-3 \xi_{0}+\alpha, \eta_{0}, 2 \alpha \xi_{0}-3 \xi_{0}^{2}+\left(X_{0}-\alpha^{2}\right) / 3\right] \in K$.

Moreover, it is evident that the mapping $\psi: L \rightarrow K$ defined by

$$
\psi\left(\xi_{0}, \eta_{0}\right)=\left[-3 \xi_{0}+\alpha, \eta_{0}, 2 \alpha \xi_{0}-3 \xi_{0}^{2}+\left(X_{0}-\alpha^{2}\right) / 3\right]
$$

is bijective. The proof is complete. 
Example 6.4. Let $D=-87$. Then $\left[X_{0}, Y_{0}\right]=[-320,11008] \in \mathcal{E}_{-87}$, and for $\alpha=1$, we have $3 \alpha X_{0}+Y_{0}-4 \alpha^{3}=10044 \equiv 0(\bmod 27)$. By Theorem 6.3 the set $K$ of all integer solutions of the system $R^{2}+3 T=-320$ and $R^{3}-9 R T+108 S^{2}=11008$ can be obtained using the set $L$ of all integer solutions of the elliptic equation $\eta^{2}=\xi^{3}-\xi^{2}+27 \xi+93$. Since $L=\{[-1, \pm 8],[7, \pm 24],[11, \pm 40],[239, \pm 3688]\}, 6.96$ yields

$$
K=\{[4, \pm 8,-112],[-20, \pm 24,-240],[-32, \pm 40,-448],[-716, \pm 3688,-170992]\} .
$$

On the other hand, by Section 4 , the set $K$ can also be determined by means of the set $E_{-87}(-320,11008)=\{[-80, \pm 1216],[-48, \pm 1728],[240, \pm 5184],[384, \pm 8640],[8592, \pm 796608]\}$ of all integer solutions of the elliptic equation $\eta^{2}=\xi^{3}+34560 \xi+4755456$.

Remark 6.5. If $\left[X_{0}, Y_{0}\right] \in \mathcal{O}_{D}$, then $X_{0}-4 \alpha^{2} \not \equiv 0(\bmod 12)$ for any $\alpha \in\{0,1,2\}$. On the other hand, by examples, it can be proved that both cases (i) and (ii) can occur:

$$
\text { (i) } 3 \alpha X_{0}+Y_{0}-4 \alpha^{3} \not \equiv 0(\bmod 108), \quad \text { (ii) } 3 \alpha X_{0}+Y_{0}-4 \alpha^{3} \equiv 0 \quad(\bmod 108) \text {. }
$$

(i) If $D=-23$, then $\left[X_{0}, Y_{0}\right]=[489,12555] \in \mathcal{O}_{-23}$, and for any $\alpha \in\{0,1,2\}$, we have $3 \alpha X_{0}+Y_{0}-4 \alpha^{3} \not \equiv 0(\bmod 108)$.

(ii) If $D=-107$, then $\left[X_{0}, Y_{0}\right]=[9241,888445] \in \mathcal{O}_{107}$, and for $\alpha=1$, we get $3 \alpha X_{0}+Y_{0}-4 \alpha^{3}=$ $916164 \equiv 0(\bmod 108)$.

\section{Some conjectures related to Mordell's equation}

Let $0 \neq D \in \mathbb{Z}$ and let

$$
\mu_{D}=\left\{\begin{array}{lll}
0 & \text { if } \quad M_{D}=\emptyset \\
1 & \text { if } \quad M_{D} \neq \emptyset
\end{array}\right.
$$

Next, for any positive integer $n$, put

$$
\sigma_{n}=\sum_{D=1}^{n} \mu_{D} \quad \text { and } \quad \sigma_{-n}=\sum_{D=-1}^{-n} \mu_{D} .
$$

Computer investigation of the values $\sigma_{n}$ and $\sigma_{-n}$ for $n \leq 1000$ yields

$$
\frac{\sigma_{1000}}{1000}=\frac{280}{1000}=0.280, \quad \frac{\sigma_{-1000}}{1000}=\frac{426}{1000}=0.426, \quad \frac{\sigma_{1000}}{\sigma_{-1000}}=\frac{280}{426} \approx 0.657 .
$$

Hence, the following conjectures can be made:

$$
\lim _{n \rightarrow \infty} \frac{\sigma_{n}}{n}=\frac{2}{7} \approx 0.286, \quad \lim _{n \rightarrow \infty} \frac{\sigma_{-n}}{n}=\frac{3}{7} \approx 0.429, \quad \lim _{n \rightarrow \infty} \frac{\sigma_{n}}{\sigma_{-n}}=\frac{2}{3} \approx 0.667 .
$$

The conjectures (7.1) lead to another interesting question, namely, whether some similar hypotheses can also be stated for the case of general Mordell's equation. It is clear that, to formulate such hypotheses, much computation will be needed.

Thanks to the computations made by M. A. Bennett and A. Ghadermarzi 11, all integer solutions of $Y^{2}=X^{3}+k$ are determined for any $0 \neq|k| \leq 10^{7}$. Based on their results, some new conjectures can be formulated. The following notations will be useful.

For $0 \neq k \in \mathbb{Z}$, let $M(k)$ denote the set of all integer solutions of $Y^{2}=X^{3}+k$, and let

$$
\mu(k)=\left\{\begin{array}{lll}
0 & \text { if } & M(k)=\emptyset \\
1 & \text { if } & M(k) \neq \emptyset
\end{array}\right.
$$


Next, for any positive integer $n$, put

$$
\sigma(n)=\sum_{k=1}^{n} \mu(k) \quad \text { and } \quad \sigma(-n)=\sum_{k=-1}^{n} \mu(-k) .
$$

By inspecting Table 1 and Table 2 in [1: pp. 642-643], we get

$$
\frac{\sigma\left(10^{7}\right)}{10^{7}}=\frac{1332934}{10^{7}} \approx 0.133, \quad \frac{\sigma\left(-10^{7}\right)}{10^{7}}=\frac{834604}{10^{7}} \approx 0.083, \quad \frac{\sigma\left(-10^{7}\right)}{\sigma\left(10^{7}\right)}=\frac{834604}{1332934} \approx 0.626 .
$$

Hence, the following conjectures can be made:

$$
\lim _{n \rightarrow \infty} \frac{\sigma(n)}{n}=\frac{2}{15}=0.1 \overline{3}, \quad \lim _{n \rightarrow \infty} \frac{\sigma(-n)}{n}=\frac{1}{12}=0.08 \overline{3}, \quad \lim _{n \rightarrow \infty} \frac{\sigma(-n)}{\sigma(n)}=\frac{5}{8}=0.625 .
$$

Our surmises can be proposed to the reader as Problem 7.1

Problem 7.1. Prove or disprove 77.1 and 7.2 .

\section{Conclusion}

The results presented in this paper make it possible to determine the set $Q_{D}$ of all monic quartic polynomials $x^{4}+a x^{3}+b x^{2}+c x+d \in \mathbb{Z}[x]$ with a given discriminant $0 \neq D \in \mathbb{Z}$. That provides an opportunity to study the following problem: Establish a general method of deciding in a finite number of steps whether, for a given $0 \neq D \in \mathbb{Z}$, the following statement holds: Let $p$ be an arbitrary prime. Then all polynomials in $Q_{D}$ have the same type of factorization over the Galois field $\mathbb{F}_{p}$. The validity of an analogous statement for the case of cubic polynomials has been recently examined in 10 -15 with relatively closed results obtained.

Acknowledgement. The author thanks the anonymous referee for carefully reading the manuscript.

\section{REFERENCES}

[1] BEnNeTT, M. A.-GHADERMARZI, A.: Mordell's equation: a classical approach, LMS J. Comput. Math. 18.1 (2015), 633-646.

[2] DICKSON, L. E.: History of the Theory of Numbers, Vol. II, Chelsea, New York, 1952.

[3] EVERTSE, J. H.-GYÖRY, K.: Discriminant Equations in Diophantine Number Theory, Cambridge University Press, Cambridge, 2017.

[4] HARDY, G. H.-WRIGHT, E. M.: An Introduction to the Theory of Numbers, 6th ed., Oxford University Press, New York, 2008.

[5] HEMER, O.: On the Diophantine Equation $y^{2}-k=x^{3}$, Doctoral Dissertation, Uppsala (1952).

[6] GAUTHIER, S.-LÊ, F.: On the youthful writings of Louis J. Mordell on the Diophantine equation $y^{2}-k=x^{3}$, Archive for History of Exact Sciences 73 (2019), 427-468.

[7] GEBEL, J.-PETHÖ, A.-ZIMMER, G. H.: On Mordell's equation, Compos. Math. 110 (1998), 335-367.

[8] GYÖRY, K.: Sur les polynômes à coefficients entiers et de discriminant donné, Acta Arith. 23 (1973), 419-426.

[9] GYÖRY, K.: Polynomials and binary forms with given discriminant, Publ. Math. Debrecen 69(4) (2006), 473-499.

[10] KLAŠKA, J.-SKULA, L.: Mordell's equation and the Tribonacci family, Fibonacci Quart. 49(4) (2011), 310-319.

[11] KLAS̆KA, J.-SKULA, L.: Law of inertia for the factorization of cubic polynomials - the real case, Util. Math. 102 (2017), 39-50.

[12] KLAŠKA, J.-SKULA, L.: Law of inertia for the factorization of cubic polynomials - the imaginary case, Util. Math. 103 (2017), 99-109. 


\section{JIŘÍ KLAŠKA}

[13] KLAS̆KA, J.-SKULA, L.: Law of inertia for the factorization of cubic polynomials - the case of discriminants divisible by three, Math. Slovaca 66(4) (2016), 1019-1027.

[14] KLAŠKA, J.-SKULA, L.: Law of inertia for the factorization of cubic polynomials - the case of primes 2 and 3, Math. Slovaca 67(1) (2017), 71-82.

[15] KLAŠKA, J.-SKULA, L.: On the factorizations of cubic polynomials with the same discriminant modulo a prime, Math. Slovaca 68(5) (2018), 987-1000.

[16] LONDON, J.-FINKELSTEIN, M.: On Mordell's Equation $y^{2}-k=x^{3}$, Bowling Green, Ohio Bowling Green State University, 1973.

[17] MORDELL, L. J.: A statement by Fermat, Proc. Lond. Math. Soc. (2) 18 (1920), pp. v-vi.

[18] SIEGEL, C. L.: Über einige Anwendungen diophantischer Approximationen, Abh. Preuss Akad. Wiss., 1929, pp. 1-41.

[19] SMART, N. P.: The Algorithmic Resolution of Diophantine Equations, Cambridge University Press, Cambridge, 1998.

Received 16. 11. 2020

Accepted 25. 1. 2021
Institute of Mathematics Faculty of Mechanical Engineering Brno University of Technology

Technická 2

61669 Brno

CZECH REPUBLIC

E-mail: klaska@fme.vutbr.cz 